\title{
Diacronie
}

Studi di Storia Contemporanea

$\mathrm{N}^{\circ} 11,3$ | 2012

La satira fa storia. Eventi, pratiche, linguaggi

\section{Antonio Maria Morone, L'ultima colonia. Come l'Italia è tornata in Africa 1950-1960}

Michele Pandolfo

\section{Q OpenEdition \\ Journals}

\section{Edizione digitale}

URL: http://journals.openedition.org/diacronie/2728

DOI: $10.4000 /$ diacronie. 2728

ISSN: 2038-0925

Editore

Association culturelle Diacronie

Notizia bibliografica digitale

Michele Pandolfo, «Antonio Maria Morone, L'ultima colonia. Come l'Italia è tornata in Africa 1950-1960», Diacronie [Online], N 11, 3 | 2012, documento 13, Messo online il 29 octobre 2012, consultato il 24 septembre 2020. URL : http://journals.openedition.org/diacronie/2728 ; DOI : https://doi.org/10.4000/ diacronie. 2728 


\section{Diacronie}

N. 11 | 3|2012 La satira fa storia. Eventi, pratiche, linguaggi

13/

RECENSIONE:

Antonio Maria MORONE, L'ultima colonia. Come l'Italia è tornata in Africa 1950-1960, Roma-Bari, Laterza, 2011, 232 pp.

a cura di Michele PANDOLFO *

Antonio Maria Morone, docente all'Università di Pavia, ha pubblicato nel corso del 2011 il libro L'ultima colonia. Come l'Italia è tornata in Africa 1950-196o per la casa editrice Laterza. Questo lavoro è il frutto di studi e ricerche sul decennio dell'Amministrazione fiduciaria italiana sulla Somalia (Afis), un periodo lasciato ai margini dalla storiografia classica del colonialismo italiano, nonostante importanti e significative pubblicazioni siano apparse nel corso dei decenni passati sull'argomento coloniale ${ }^{1}$.

È opinione diffusa che la perdita delle colonie da parte dell'Italia sia stata un'inevitabile conseguenza politica delle numerose sconfitte militari avvenute durante la seconda guerra mondiale. In realtà la risoluzione del problema delle colonie italiane si rivelò sin dall'inizio una questione diplomatica lunga e complessa, come ci conferma

\footnotetext{
1 Anche se marginale rispetto ad altri argomenti storiografici, l'attenzione al colonialismo italiano è rappresentata da alcune importante ricerche storiche. Il padre di questi studi è considerato Angelo Del Boca con i suoi tre volumi intitolati Gli italiani in Africa Orientale, Roma-Bari, Laterza, 1976-1982, ai quali sono seguiti altri importanti testi come L'Africa nella coscienza degli italiani: miti, memorie, errori, sconfitte, Roma, Laterza, 1992; Italiani brava gente? Un mito duro a morire, Vicenza, Neri Pozza, 2005 e alcuni testi specifici riguardanti il colonialismo italiano in Libia e in Somalia. Altri storici si sono occupati di questo tema: ROCHAT, Giorgio, Il colonialismo italiano, Torino, Loescher, 1974 e LABANCA, Nicola, Oltremare: storia dell'espansione coloniale italiana, Bologna, Il Mulino, 2002. Dal punto di vista teorico e culturale segnalo invece alcuni studi che affrontano la questione postcoloniale nell'Italia contemporanea: BEN-GHIANT, Ruth, FULLER, Mia, Italian Colonialism, New York, Palgrave, 2005; PARATI, Graziella, Mediterranean Crossroads: Migration Literature in Italy, London, Associated University Presses, 1999; Id., Migration Italy. The Art of Talking Back in a Destination Culture, Toronto, University of Toronto Press, 2005; FORGACS, David, LUMLEY, Robert, Italian Cultural Studies. An Introduction, Oxford, Oxford University Press, 1996. Inoltre, in lingua italiana, ricordo la presenza dell'originale e prezioso studio di genere: STEFANI, Giulietta ( a cura di ), Colonia per maschi. Italiani in Africa Orientale: una storia di genere, Verona, Ombre corte, 2007.
} 
lo stesso Antonio Morone: «La "decolonizzazione dall'alto" degli ex possedimenti italiani assunse i connotati di un'intricata vicenda politico-diplomatica che si chiude solo dopo quasi un decennio dal crollo dell'Africa orientale italiana (Aoi) nel 1941.»². Dopo i primi tentativi di ottenere nuovamente il controllo sulla Libia o su una delle sue regioni si aprì il contenzioso per l'Eritrea, che i governi italiani neodemocratici richiesero a gran voce facendo leva sulla lunga appartenenza della colonia "primigenia" all'Italia. Ma dopo alcuni anni di trattative con i governi alleati e una serie di veti incrociati, la questione delle colonie italiane passò direttamente al giudizio dell'Assemblea Generale delle Nazione Unite che, nel 1949, essendo ormai sicura l'indipendenza della Libia e la federazione dell'Eritrea con l'Etiopia, si trovò invece a decidere il destino della Somalia. Infatti l'ultima ex colonia italiana si rivelò anche l'unica che le quattro potenze internazionali - gli Stati Uniti d'America, la Gran Bretagna, la Francia e l'Unione Sovietica - erano disposte a concedere nuovamente all'Italia democratica. Il ritorno italiano in Somalia fu però inquadrato in una cornice istituzionale del tutto nuova ed eccezionale chiamata Trusteeship System, che rappresenta il tema principale delle riflessioni storiche di Antonio Morone. Con questo strumento giuridico l'Italia si impegnava a condurre la Somalia verso l'indipendenza in un arco di tempo stabilito in dieci anni e si assumeva questa gravosa responsabilità di fronte al popolo somalo - ex colonizzato - e alla comunità internazionale. Nell'introduzione al testo, scritta dallo stesso studioso, si descrive in maniera dettagliata questa complessa fase:

In Africa la cesura storica che gli eventi bellici avrebbero potuto rappresentare cedette invece a una più lunga fine del colonialismo, che passò attraverso la propaganda colonialista degli anni Quaranta e il decennio dell'Afis. L'Italia chiese a gran voce di ritornare in colonia dopo la sconfitta in guerra e accettò il mandato di tutela sulla Somalia per conto dell'Onu, nello spirito di una prova di recupero per il passato. La più periferica delle ex colonie costituì così l'eccezione dell'eccezione: la nuova Italia venne chiamata a guidare la decolonizzazione della Somalia nella specialissima cornice del Trusteeship System. L'esperimento di un colonialismo democratico nascondeva tuttavia una serie di intrinseche contraddizioni insieme alla difficoltà di riprendere e concludere, nell'arco di appena un decennio, la costruzione di quella società moderna che proprio in Somalia era sempre rimasta, più che altrove, a uno stadio iniziale3.

\footnotetext{
2 MORONE, Antonio Maria, L'ultima colonia. Come l'Italia è tornata in Africa 1950-196o, Roma-Bari, Laterza, 2011, p. 5.

3 Ibidem, p. X.
} 
In questo delicato quadro i compiti che l'Accordo di Tutela delle Nazioni Unite affidava all'Italia sono stati analizzati da Antonio Morone in maniera puntuale e dettagliata. L'Italia si assunse l'incarico di formare la nuova classe dirigente somala creando apparati amministrativi efficienti; di garantire la costruzione dei servizi pubblici essenziali, come le scuole e gli ospedali; di formare un nuovo esercito somalo e infine, ma non ultimo punto in ordine di importanza, di cercare di costruire un'economia capace di dare lavoro e ricchezza alla futura nazione indipendente. Il compito dell'Italia non fu facile: il contesto sociale e politico somalo nel quale i funzionari italiani si trovarono a lavorare era caratterizzato da un forte sentimento anti-italiano. Infatti se da una parte vi era lo scetticismo della maggior parte dei somali che non volevano un ritorno all'amministrazione precedente, dall'altra si ponevano le resistenze dell'amministrazione militare britannica che non voleva rinunciare al proprio controllo diretto e alla propria influenza nel Corno d'Africa. Nel mezzo della disputa emersero le posizioni dei nuovi movimenti politici somali che si erano formati nel corso degli anni Quaranta: essi cercarono nella maggior parte dei casi di mantenere un atteggiamento autonomo rispetto alle posizioni dei paesi occidentali e richiesero per la Somalia un'immediata proclamazione d'indipendenza. La più forte resistenza al ritorno italiano in Somalia fu rappresentata dalla Lega dei Giovani Somali, che, soprattutto nei primi anni del protettorato, fu la vera antagonista di tutte le azioni dell'Afis. A tal proposito Antonio Morone scrive:

L’inclusione della Somali Youth League (Syl) nel sistema fiduciario si sarebbe dimostrata il vero nodo politico da sciogliere per i vertici dell'Afis. Nonostante le prove d'intesa tra Syl e Afis fossero iniziate addirittura prima del ritorno ufficiale dell'Italia in Somalia, la collaborazione vera arrivò solo più tardi con la partecipazione della Lega alle elezioni amministrative del 1954 e poi a quelle politiche del 19564 .

Il lavoro e il saggio di Antonio Morone si basano su fonti di grandissimo valore, provenienti, tra gli altri, dall'Archivio Centrale dello Stato, dagli Archivi dell'Esercito italiano e l'Archivio del Ministero degli Affari Esteri, dove sono confluiti i documenti dell'ex Ministero delle Colonie, sciolto nel 1953. Inoltre lo studioso ha consultato il Fondo Giuseppe Brusasca, conservato presso l'Archivio Storico del comune di Casale Monferrato. Antonio Morone delinea con le proprie parole la sua figura politica: «Giuseppe Brusasca, che per aver ricoperto la carica di sottosegretario agli esteri con delega all'Africa Italiana quasi senza interruzione dal 1946 al 1953 fu uno dei principali

4 Ibidem, p. 50. 
artefici della politica italiana in Africa, considerava le colonie "il problema fondamentale" della politica estera italiana insieme alla disputa con la Jugoslavia per Trieste»5.

Il mandato internazionale dell'Italia - iniziato nel 1950 - si concluse il primo luglio 1960, con sei mesi di anticipo rispetto alla sua scadenza naturale. Da quel momento l'ex Somalia italiana e l'ex Somalia britannica si unirono per dar vita alla nuova repubblica somala con capitale Mogadiscio. L'Italia ritirò i propri amministratori e funzionari, ma la sua influenza sulla Somalia e i suoi interessi in quella terra, seppur deboli e fragili, di certo non cessarono con la fine del mandato. Il lavoro di Antonio Morone risulta originale e importante principalmente per due ragioni: in primo luogo per via dall'assenza nel panorama accademico ed editoriale italiano di un adeguato approfondimento della ricerca storica sul periodo dell'Afis; il secondo per l'attenta analisi degli errori che l'amministrazione fiduciaria italiana commise durante il suo mandato, nonostante le iniziali buone intenzioni e il lodevole lavoro compiuto da alcuni funzionari meritevoli. Infatti al momento dell'indipendenza somala le fragilità politiche, economiche e culturali del nuovo Stato erano già evidenti, tanto che la democrazia parlamentare e multipartitica, così pazientemente costruita sul modello italiano, resistette solo nove anni - cioè sino al 1969 - prima dell'avvento della dittatura del generale Siad Barre.

5 Ibidem, pp. 6-7. 


\section{* L'autore}

Michele Pandolfo ha conseguito la Laurea Specialistica in Filologia Moderna; è oggi dottorando in Storia, Culture e Strutture delle Aree di Frontiera presso l'Università di Udine, in cotutela con l'Université Paul Valery di Montpellier.

URL: <www.studistorici.com/autori/\#Pandolfo >

\section{Per citare questo articolo:}

PANDOLFO, Michele, «Recensione: Antonio Maria MORONE, L'ultima colonia. Come l'ttalia è tornata in Africa 19501960, Bari-Roma, Laterza, 2011, 232 pp.», Diacronie. Studi di Storia Contemporanea: La satira fa storia. Eventi, pratiche, linguaggi, 29/10/2012,

URL:< http://www.studistorici.com/2012/10/29/pandolfo_numero_11/ >

Diacronie Studi di Storia Contemporanea $\widehat{w}$ ww.diacronie.it

Risorsa digitale indipendente a carattere storiografico. Uscita trimestrale.

redazione.diacronie@hotmail.it

Comitato di redazione: Marco Abram - Giampaolo Amodei - Jacopo Bassi - Luca Bufarale - Gianluca Canè - Alessandro Cattunar - Alice De Rensis - Barbara Galimberti - Deborah Paci - Fausto Pietrancosta - Matteo Tomasoni - Luca Zuccolo

Diritti: gli articoli di Diacronie. Studi di Storia Contemporanea sono pubblicati sotto licenza Creative Commons 2.5

Possono essere riprodotti a patto di non modificarne i contenuti e di non usarli per fini commerciali. La citazione di

estratti è comunque sempre autorizzata, nei limiti previsti dalla legge. 